\title{
Semiconductor Ridge Microcavities Generating Counterpropagating Entangled Photons
}

\author{
Xavier Caillet, Adeline Orieux, Ivan Favero, Giuseppe Leo and Sara Ducci \\ Laboratoire Matériaux et Phénomènes Quantiques UMR 7162 \\ Université Paris Diderot Paris 7- CNRS
}

France

\section{Introduction}

In the last 25 years entangled photon pairs have been used first to test the foundations of quantum mechanics and then as building blocks in quantum information protocols : from the demonstration of the violation of Bell inequalities (Aspect et al., 1982; Tittel et al., 1998; Weihs et al., 1998) to the recent experiments in the domain of quantum-key distribution (Gisin et al., 2002), quantum computing (Deutch \& Ekert, 1998; Wahther et al., 2005), teleportation (Bouwmeester et al., 1997) and absolute metrology (Migdall, 1999; Sergienko \& Jaeger, 2003).

The first process used to produce entangled two-photon states have been atomic radiative cascades (Aspect et al., 1982) and parametric fluorescence in birefringent dielectric materials (Kwiat et al., 1985).

In order to achieve a good source, high collection efficiency is a key element because it affects the number of available photon pairs per unit time, and also because the presence of single photons having lost their twin is a source of noise in the detection. In this context we can mention several demonstrations of twin-photon generation based on either parametric down-conversion in periodically poled dielectric waveguides (Tanzilli et al., 2001; Banaszek et al., 2001) or four-wave mixing in optical fibers (Wang et al., 2001; Rarity et al. 2005; Fan \& Migdall, 2007).

An attractive alternative is provided by semiconductor materials, which exhibit a huge potential in terms of integration of novel optoelectronic devices. The first semiconductor source of entangled photons was based on the bi-exciton cascade of a quantum dot (Stevenson et al., 2006). With respect to this technique, parametric generation in semiconductor waveguides allows room-temperature operation and a high directionality of the emission, which dramatically enhances the collection efficiency. Several phase-matching schemes have been demonstrated in these systems (Ducci et al., 2005): form birefringence, modal phase matching, counterpropagating phase matching. In particular, the last one has attracted a deal of attention because of its unusual flexibility in the control of the quantum properties of the emitted photons (Walton et al., 2003; Walton et al., 2004; Perina, 2008).

Here we present a semiconductor ridge microcavity emitting counterpropagating entangled photons; in Section 2 we explain the working principle of this device giving the details of the phase matching scheme and the effects of the microcavity. Section 3 is devoted to the

Source: Advances in Lasers and Electro Optics, Book edited by: Nelson Costa and Adolfo Cartaxo,

ISBN 978-953-307-088-9, pp. 838, April 2010, INTECH, Croatia, downloaded from SCIYO.COM 
description of the quantum properties of the generated two-photon state, which illustrate the versatility of the source. The experimental results of Surface Emitted Second Harmonic Generation and Parametric Fluorescence are presented in Section 4. Finally, we draw our conclusions in Section 5.

\section{Working principle and microcavity effect}

The twin-photon source presented here is a multilayer AlGaAs waveguide designed to allow a counterpropagating phase matching scheme. The lateral confinement is provided by a wet-etched ridge. In this geometry (Figure 1), a pump field $(775 \mathrm{~nm})$ impinges on top of the waveguide generating two counterpropagating, orthogonally polarized waveguided twin photons $(\sim 1550 \mathrm{~nm})$ through parametric down-conversion. The frequencies of the emitted fields are fixed by the energy $\left(\omega_{p}=\omega_{s}+\omega_{\imath}\right)$ and momentum $\left(k_{p} \sin \theta=n_{s} k_{s}-n_{i} k_{i}\right)$ conservation, where $\omega_{p}, \omega_{s}$ and $\omega_{i}\left(k_{p}, k_{s}\right.$ and $\left.k_{i}\right)$ are the frequencies (wavevectors) of pump, idler and signal; $\theta$ is the angle of incidence of the pump beam, and $n_{s}$ and $n_{i}$ are the effective indices of the signal and idler modes. Momentum conservation in the epitaxial direction is satisfied by alternating AlGaAs layers with different $\mathrm{Al}$ concentration (having nonlinear coefficients $d_{14}$ as different as possible) to obtain a Quasi Phase Matching (QPM).

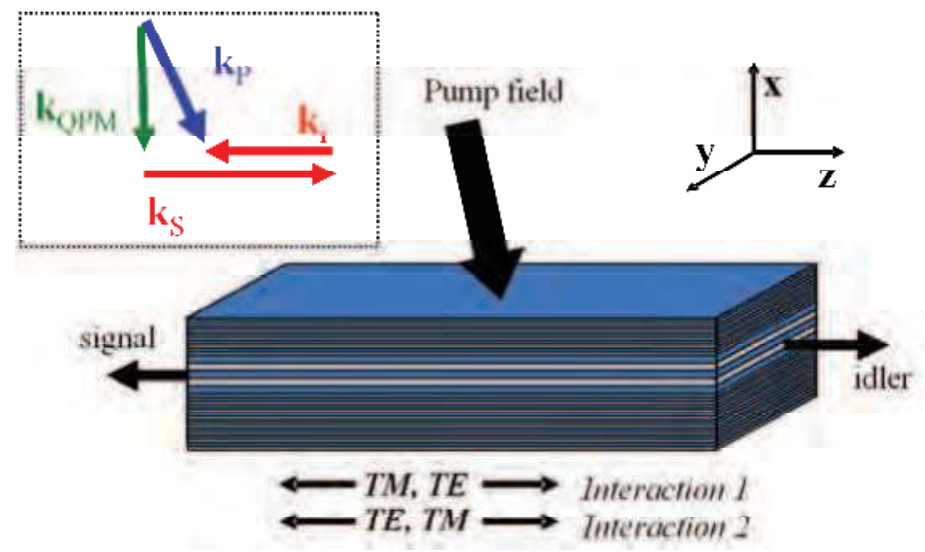

Fig. 1. Counterpropagating phase-matching scheme. Phase matching is automatically obtained in the $z$ direction; Quasi Phase Matching (QPM) is provided by a periodic modulation of the nonlinear susceptibility tensor in the epitaxial direction in the core of the waveguide.

As a consequence of the different propagation directions for the signal and the idler, there are two simultaneously phase-matched processes: one where the signal is TE polarized and the idler is TM polarized, and the other where the signal is TM polarized and the idler is TE polarized. These two processes are expected to exhibit different characteristics because of the $z \rightarrow-z$ symmetry breaking exerted by the pump beam for $\theta \neq 0$. In the following, subscripts 1 and 2 will refer to these two processes. The central frequencies for the signal and the idler (corresponding to phase matching) are determined through the conservation of energy and momentum in the $\mathrm{z}$ direction, giving: 


$$
\begin{aligned}
& \left\{\begin{array}{l}
\omega_{p}=\omega_{s}+\omega_{i} \\
\omega_{p} \sin \theta=\omega_{s} n_{\mathrm{TE}}\left(\omega_{s}\right)-\omega_{i} n_{\mathrm{TM}}\left(\omega_{i}\right)
\end{array}\right. \\
& \left\{\begin{array}{l}
\omega_{p}=\omega_{s}+\omega_{i} \\
\omega_{p} \sin \theta=\omega_{s} n_{\mathrm{TM}}\left(\omega_{s}\right)-\omega_{i} n_{\mathrm{TE}}\left(\omega_{i}\right)
\end{array}\right.
\end{aligned}
$$

where $n_{T E}$ and $n_{T M}$ are the effective indices of the guided modes.

Several advantages derive from such a geometry, in the aim of fabricating a source for quantum information: absence of the pump beam in the guided direction, automatic separation of the down-converted photons, and possibility of direct coupling into two optical fibers through standard pig-tailing process.

A preliminary demonstration of a twin-photon source based on this principle has been reported by (Lanco et al., 2006); however the performances of that device were affected by low conversion efficiency and low signal/noise ratio due to photo-luminescence from the substrate.

An interesting possibility to improve the source consists in the addition of Distributed Bragg Reflectors (DBRs) on top of and under the waveguide, in order to create a microcavity for the pump beam (Andronico et al., 2008). In this case, at resonance wavelength, a nearly standing wave is obtained inside the cavity, whose amplitude can be largely greater than outside (see Figure 2).

In the case of the waveguide without microcavity presented above, the guided field amplitude of the pump beam can be roughly estimated using the Fresnel equations. Assuming, for the sake of simplicity, a homogeneous waveguide with refractive index $n_{\text {guide }}$ and air as incident medium, the relation between the internal and external pump field is $E_{p}{ }^{i n t}$ $=t E_{p}$ ext , with $|t|=2 /\left(1+n_{\text {guide }}\right)$.

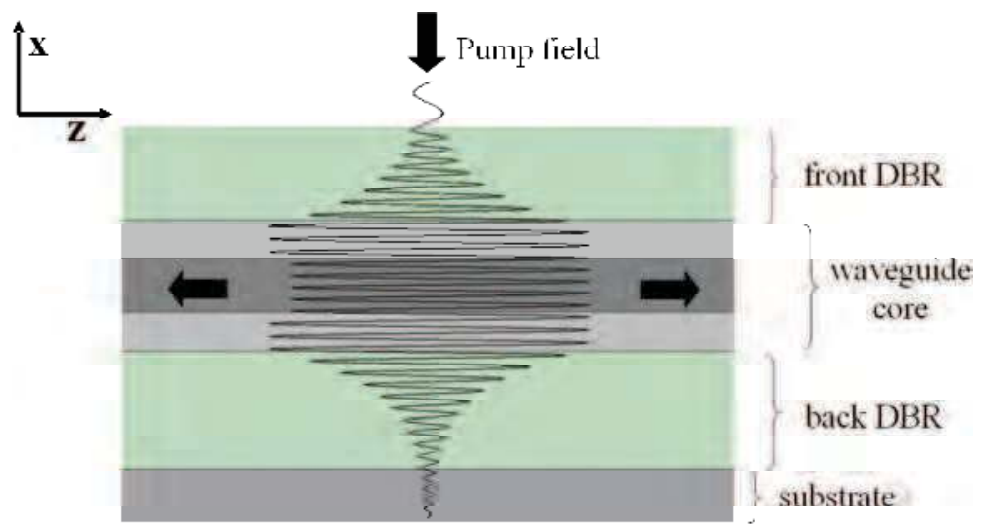

Fig. 2. View in the (xz) plane of the waveguide with the addition of the DBRs. In black is plotted the pump beam profile within the structure at cavity resonance. The field enhancement induces a strong improvement of the conversion efficiency.

In the case of the ridge microcavity showed in Figure 2, we can use the transfer matrix method to compute the amplitude of the standing wave inside the cavity. This is found to be: 


$$
\frac{E_{p}^{\mathrm{int}}}{E_{p}^{\text {ext }}}=2 \sqrt{\frac{2 F}{\pi\left(1+\left|t_{2} / t_{1}\right|^{2}\right)}}
$$

Here, $F$ is the finesse of the cavity, and $t_{i}$ are the transmission coefficients associated to the front $(i=1)$ and back $(i=2)$ mirrors. It can be shown that the conversion efficiency enhancement factor due to the addition of the microcavity is:

$$
\frac{\eta_{\text {cavity }}}{\eta_{0}}=\frac{2\left(1+n_{\text {guide }}\right)^{2}}{\pi n_{\text {guide }}} \frac{F}{\left(1+\left|t_{2} / t_{1}\right|^{2}\right)}
$$

This expression indicates how to optimize the conversion efficiency.

Firstly, the ratio $\left|t_{2} / t_{1}\right|$ has to be minimized, reflecting the obvious fact that the front mirror should be less reflective than the back one, in order to let the light enter the microcavity.

Secondly, a maximum finesse is desired. It is interesting, for the rest of the discussion, to introduce the quality factor of the cavity: $Q=m_{\text {eff }} F$. Here, $m_{\text {eff }}$ is the effective order of the cavity, given by $m_{\text {eff }}=m+m_{0}$, where $\mathrm{m}$ is the cavity order and $\mathrm{m}_{0}$ accounts for the penetration of the field in the DBR. For a $\lambda / 4$ DBR stack, $m_{0}=n_{H} /\left(n_{H}-n_{L}\right)$, where $n_{H}$ and $n_{L}$ are the refractive indices of the high- and low-index materials. Equation (3) shows that the higher the quality factor, the higher the efficiency.

However, a practical limitation on $Q$ stems from technical reasons. Firstly, the large amount of energy stored in the cavity can possibly induce thermorefractive effects, leading to undesirably bistable devices. Moreover, the inhomogeneity of the sample inherent to the growth process induces a variation of the cavity resonance wavelength $\lambda$ along the direction of the waveguide. To obtain an efficient interaction throughout the structure, this variation has to be smaller than the mean cavity linewidth $\delta \lambda$. This, in return, sets an upper limit to the desired quality factor, expressed as $\mathrm{Q}=\lambda / \delta \lambda$.

\section{Quantum properties of the two-photon state}

In this Section we derive the quantum state of the photon pair emitted by our source, showing how the pump beam parameters, in particular its shape and bandwidth, can be used to control the degree of frequency correlation.

In a typical down-conversion experiment using a narrow-band pump beam, the twin photons have perfectly anti-correlated frequencies (as the sum of their energies is fixed by the pump photon energy). However the use of short pump pulses relaxes the strict frequency anti-correlation of the generated photons and open the possibility to generate frequency correlated or frequency uncorrelated states.

The Hamiltonian describing the parametric down conversion process is:

$$
H(t)=\int d V\left(\chi_{e f f}^{(2)}(r) E_{p}^{(+)}(r, t) E_{s}^{(-)}(r, t) E_{i}^{(-)}(r, t)+h . c .\right)
$$

where $\chi_{\text {eff }}^{(2)}$ is the effective electric susceptibility tensor (periodically modulated along the epitaxial axis $(x)$ to provide quasi phase matching as described in the previous section), $E_{p}^{(+)}$ the positive-frequency part of the pump beam electric-field operator, $E_{s}^{(-)}$and $E_{i}^{(-)}$the negative-frequency part of the signal and idler electric-field operators. The symbol h.c. 
stands for hermitian conjugate term. The integration is performed over the interaction volume which, in our case, is the illuminated portion of the waveguide.

The suitability of a photon pair source for a given quantum optics application largely depends on the joint spectral intensity (JSI) of the generated photons, $S\left(\omega_{s}, \omega_{i}\right)$, defined as the probability that the signal (idler) photon frequency is $\omega_{s},(\omega)$. In the following we make explicit the link between the JSI and the characteristics of the pump beam in order to clarify the physical parameters that can be used to tailor the two-photon state.

A classical pump field on the air side of the air/semiconductor interface is given by:

$$
E_{p}(z, t)=\int d \omega \tilde{E}(\omega) A(z) e^{i[k(\omega) \sin \theta(\omega) z-\omega t]}
$$

where we have neglected the pump variation along the $y$ axis.

In this expression: the spatial profile $A(z)$ can be engineered through linear optics; $\tilde{E}(\omega)$ is the spectral distribution, characterized by a center frequency $\omega_{p}$ and a bandwidth $\sigma_{p} ; \theta(\omega)$ is the angle of incidence of the $\omega$ component of the field, which can be engineered through a dispersive element, such as a quartz wedge or a diffraction grating.

Following the derivation of (Grice et al., 2001) and assuming incident signal and idler fields in the vacuum states, the first-order perturbation solution of the Schrödinger equation using Equation 4 gives the generated two photon state:

$$
|\psi\rangle=\propto \iint d \omega_{s} d \omega_{i} f\left(\omega_{s}, \omega_{i}\right) \hat{a}_{s}^{+}\left(\omega_{s}\right) \hat{a}_{i}^{+}\left(\omega_{i}\right)|v a c\rangle
$$

Here $\hat{a}_{s}^{+}$and $\hat{a}_{i}^{+}$are the photon creation operators for the signal and idler beams, and the probability amplitude $f\left(\omega_{s}, \omega_{\imath}\right)$ is given by:

$$
f\left(\omega_{s}, \omega_{i}\right) \propto \tilde{E}\left(\omega_{s}+\omega_{i}\right) \phi\left(\omega_{s}, \omega_{i}\right)
$$

with the phase-matching function:

$$
\phi\left(\omega_{s}, \omega_{i}\right)=\int d z \chi_{\text {eff }}^{(2)}(z) A(z) e^{i \Delta k\left(\omega_{s}, \omega_{i}\right) z}
$$

$\Delta k\left(\omega_{s}, \omega_{i}\right)=k\left(\omega_{s}+\omega_{i}\right) \sin \theta\left(\omega_{s}+\omega_{i}\right)-\beta_{s}\left(\omega_{s}\right)+\beta_{i}\left(\omega_{i}\right)$ being the $z$ component of the wave vector mismatch, and $\beta_{s, i}(\omega)$ the effective propagation constant of the signal and idler photon respectively.

As the JSI is related to the two-photon probability amplitude by the simple relation $S\left(\omega_{s}, \omega_{i}\right)=\left|f\left(\omega_{s}, \omega_{i}\right)\right|^{2}$, it is clear that the pump beam characteristics are a useful means to control the JSI.

The possibilities to tailor the JSI offered by the counterpropagating geometry are numerous and a complete review is beyond the scope of this chapter; let us focus here on some simple examples of pump-beam shaping that are sufficient to provide a large class of JSI. In particular we will show that the pump spectral profile can be used as the only parameter to generate generalized frequency-correlated states. Different techniques and geometries have been reported in the purpose of generating frequency-uncorrelated photon pairs in guidedwave co-propagating schemes. All these techniques require to fulfil particular conditions on group-velocity matching and hence on material dispersion. Here we show that there is no need for such requirements for our source: frequency uncorrelated, as well as frequency 
correlated and anti-correlated states, are easily obtained by properly choosing the pump pulse duration.

In order to investigate a feasible experimental situation, let us consider a Gaussian spectral pump distribution:

$$
\tilde{E}(\omega) \propto \exp \left[-\left(\frac{\omega-\omega_{p}}{\sigma_{p}}\right)^{2}\right]
$$

Moreover, let us assume a uniform profile of the pump field along the waveguide, and a fixed angle of incidence $\theta$ (independent of the pump frequency components). Under these conditions, we obtain:

$$
f\left(\omega_{s}, \omega_{i}\right) \propto \exp \left[-\left(\frac{\omega_{s}+\omega_{i}-\omega_{p}}{\sigma_{p}}\right)^{2}\right] \phi\left(\omega_{s}, \omega_{i}\right)
$$

with

$$
\phi\left(\omega_{s}, \omega_{i}\right)=\operatorname{sinc}\left(\frac{L}{2}\left(\Delta k\left(\omega_{s}, \omega_{i}\right)\right)\right) \exp \left(-i \frac{L}{2}\left(\Delta k\left(\omega_{s}, \omega_{i}\right)\right)\right)
$$

The probability amplitude of the two-photon state is thus the product of two functions: one depending on the pump spectral properties, and the other, the phase matching function $\phi$, on the spatial pump properties. In the following we will consider a perfect phase matching situation with $\omega_{s}^{0}$ and $\omega_{i}^{0}$ be the signal and idler frequencies. The elimination of frequency correlations can easily be shown if we approximate the $\operatorname{sinc}(x / 2)$ function by the Gaussian $\exp \left(-\gamma x^{2}\right)$, with $\gamma=0.0482$ (this value of $\gamma$ is chosen to obtain the same width at half maximum for the two functions). By introducing the reciprocal group velocities:

$$
\beta_{s, i}^{\prime}=\left.\frac{d \beta_{s, i}(\omega)}{d \omega}\right|_{\omega=\omega_{s, i}^{0}} \text { and } k^{\prime}=\left.\frac{k(\omega)}{d \omega}\right|_{\omega=\omega_{p}}=\frac{1}{c}
$$

and performing a first-order development of the phase-matching function we obtain for the joint spectral density:

$$
\begin{aligned}
S\left(\omega_{s}, \omega_{i}\right) \propto & \exp \left[-2\left(\omega_{s}-\omega_{s}^{0}\right)^{2}\left(\frac{1}{\sigma_{p}^{2}}+\gamma L^{2}\left(k^{\prime} \sin \theta-\beta_{s}^{\prime}\right)^{2}\right)\right] \\
\times & \exp \left[-2\left(\omega_{i}-\omega_{i}^{0}\right)^{2}\left(\frac{1}{\sigma_{p}^{2}}+\gamma L^{2}\left(k^{\prime} \sin \theta+\beta_{i}^{\prime}\right)^{2}\right)\right] \\
& \times \exp \left[-4\left(\omega_{s}-\omega_{s}^{0}\right)\left(\omega_{i}-\omega_{i}^{0}\right)\left(\frac{1}{\sigma_{p}^{2}}+\gamma L^{2}\left(k^{\prime} \sin \theta-\beta_{s}^{\prime}\right)\left(k^{\prime} \sin \theta+\beta_{i}^{\prime}\right)\right)\right]
\end{aligned}
$$

In this expression the condition:

$$
\frac{1}{\sigma^{2}}+\gamma L^{2}\left(k^{\prime} \sin \theta-\beta_{s}^{\prime}\right)\left(k^{\prime} \sin \theta+\beta_{i}^{\prime}\right)=0
$$


allows the factorization of the JSI and thus the generation of a frequency-uncorrelated state. We notice that this condition can always be fulfilled in our geometry by a proper choice of the pump pulse duration, Figure 3 reports numerical simulations of the JSI obtained for three different sets of waveguide lengths and pump pulse durations. In a 2D representation with signal and idler frequency as coordinates, the JSI level curves of ideal frequency correlated and anti-correlated states are segments parallel to the $\omega_{3}+\omega_{l}$ and $\omega_{3}-\omega_{1}$ directions respectively. When the correlation is not perfect, these segments become ellipses, the higher the degree of correlation the higher the eccentricity. The JSI level curves of uncorrelated frequency states are ellipses with axes in the $\omega_{s}$ and $\omega_{i}$ directions; in this case the JSI can be factorized as a product of independent probabilities for the signal and idler photons, $S\left(\omega_{s}, \omega_{i}\right)=p\left(\omega_{s}\right) p\left(\omega_{i}\right)$ : the measurement of the frequency of one of the emitted photons does not yield any information about the frequency of the other one.

The ellipse in Figure 3 (left) represents a state with frequency anti-correlation: the length of its major axis is set by the phase matching function, which depends on the waveguide length $L$. The anticorrelation can readily be maximized as the ellipse minor axis is made arbitrarily small by increasing the pump pulse duration $(\sigma \rightarrow 0)$.

A frequency correlated state is depicted in Figure 3 (centre): here the length of the major axis is set by the pump pulse duration, whereas that of the minor axis depends on the phasematching function and can be reduced by increasing the waveguide length. It is worth noting that the counterpropagating geometry allows an easy generation of such a state thanks to the sharply peaked phase-matching versus $\omega_{s}-\omega_{1}$. We stress that alternative geometries require more stringent conditions, on either group velocities or other pump properties (extended phase matching (Giovannetti et al., 2002), achromatic phase matching (Torres et al., 2005)).

Finally a frequency uncorrelated case is shown in Figure 3 (right). Here $\sigma$ and $L$ have been chosen to equal the major and minor axes length of the ellipse. The JSI appears as a circle and is a special case of frequency uncorrelated state with equal individual bandwidth of the generated photons.

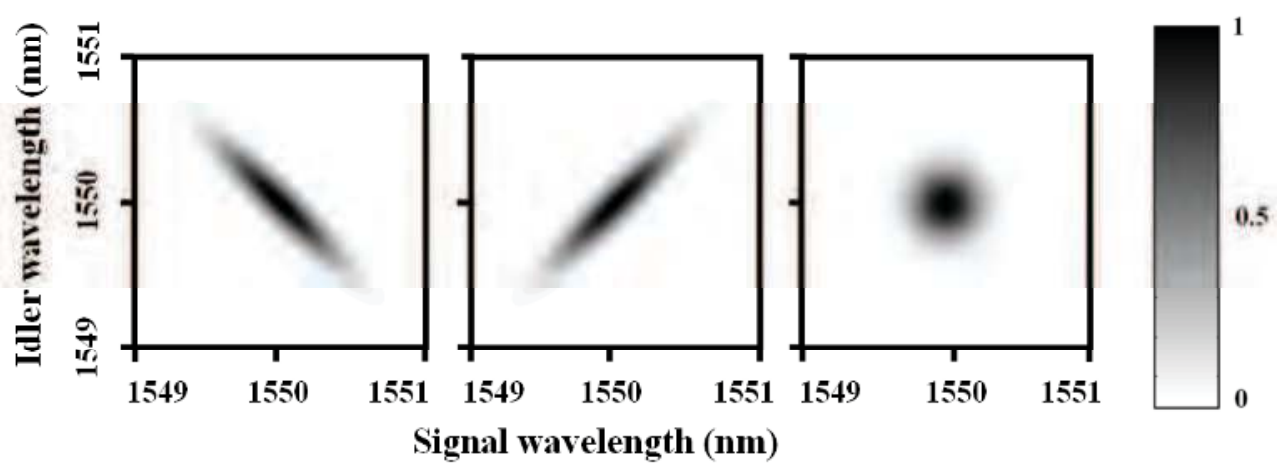

Fig. 3. Two-dimensional representation of the two-photon joint spectral intensity for three pumping configurations. Here $\mathrm{L}$ is the waveguide length (completely illuminated) and $\tau$ the pulse duration of the pump beam: (a) $\mathrm{L}=0,22 \mathrm{~mm}$ and $\tau=11 \mathrm{ps}$ (b) $\mathrm{L}=1,1 \mathrm{~mm}$ and $\tau=2.2 \mathrm{ps \text {, }}$ (c) $\mathrm{L}=0.5 \mathrm{~mm}$ and $\tau=5 \mathrm{ps}$. These configurations correspond to an anti-correlated, correlated and uncorrelated state, respectively. 
In order to quantify the separability of the generated state one has to perform a Schmidt decomposition (Law et al., 2000) of the two-photon probability amplitude $f\left(\omega_{s}, \omega_{i}\right)$, i.e. a basis transformation into a set of orthonormal Schmidt modes $\left\{\psi_{n}, \varphi_{n}\right\}$.

The function $f$ is then expressed as:

$$
f\left(\omega_{s}, \omega_{i}\right)=\sum_{n} \sqrt{\lambda_{n}} \psi_{n}\left(\omega_{s}\right) \varphi_{n}\left(\omega_{i}\right)
$$

where $\lambda_{n}, \varphi_{n}, \psi_{n}$ are solutions of the eigenvalue problem:

$$
\begin{gathered}
\iint f\left(\omega_{s}, \omega^{\prime \prime}\right) f^{*}\left(\omega^{\prime}, \omega^{\prime \prime}\right) d \omega^{\prime \prime} \psi_{n}\left(\omega^{\prime}\right) d \omega^{\prime}=\lambda_{n} \psi_{n}\left(\omega_{s}\right) \\
\iint f\left(\omega^{\prime \prime}, \omega_{i}\right) f^{*}\left(\omega^{\prime \prime}, \omega^{\prime}\right) d \omega^{\prime \prime} \varphi_{n}\left(\omega^{\prime}\right) d \omega^{\prime}=\lambda_{n} \varphi_{n}\left(\omega_{i}\right)
\end{gathered}
$$

with

$$
\sum \lambda_{n}=1
$$

If the sum in expression (15) has a unique term, $\mathrm{f}$ is factorizable and the photons of the pair are not entangled. If the sum in expression (15) contains a large number of terms the state of the pair is strongly entangled. The measure of the correlation degree is provided by the entropy $S$ :

$$
S=-\sum_{n=1}^{N} \lambda_{n} \log _{2}\left(\lambda_{n}\right)
$$

where $N$ is the number of Schmidt modes used in the decomposition. $S$ is equal to zero for a non entangled state and is the more important the more the state is entangled. To give an example, Figure 4 reports the Schmidt decomposition of a frequency correlated and a frequency anti-correlated state.

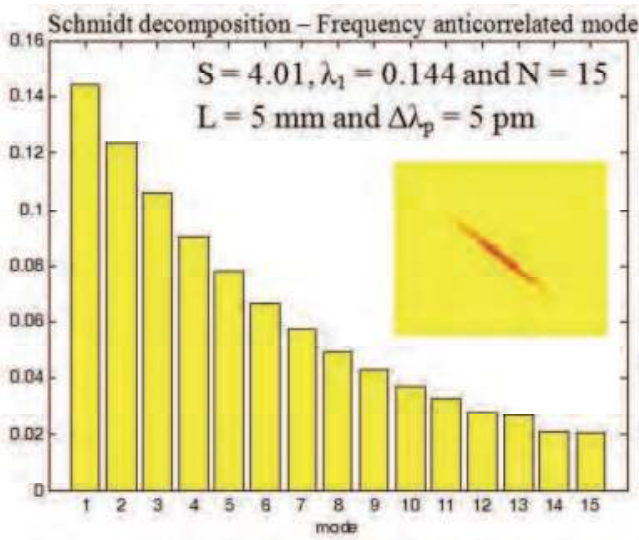

Schmidt decomposition - Frequency correlated mode

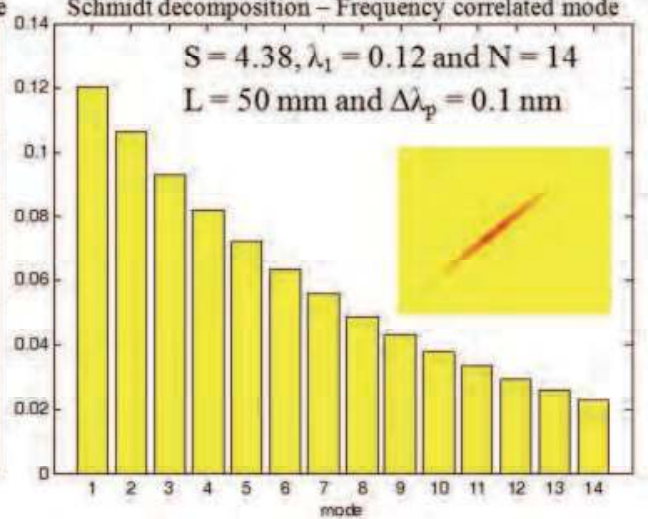

Fig. 4. Schmidt decomposition for a frequency anti-correlated and a frequency correlated mode. $L$ is the length of the guide (completely illuminated) and $\Delta \lambda_{p}$ the spectral width of the pump beam. 
The relative simplicity of frequency entanglement control by a suitable choice of the pump bandwidth is peculiar of the counterpropagating geometry. Further theoretical developments have been made towards the generation of photon pairs with arbitrary joint spectrum (Walton et al., 2004; Perina, 2008). In particular, a more refined shaping of the pump beam using achromatic phase matching (i.e. allowing the angle $\theta$ to vary with $\omega$ ) has been shown to generate uncorrelated twin photons with independent spectral bandwidth (Walton et al., 2004).

\section{Experimental results}

\subsection{Surface emitted second harmonic generation}

A practical mean to characterize the efficiency enhancement due to the integration of a vertical microcavity for the pump beam is Surface Emitted Second Harmonic Generation (SESHG) (Caillet et al., 2009). SESHG, which was first demonstrated in 1979 (Normandin \& Stegeman, 1979), is the reverse of SPDC at degeneracy $\left(\omega_{s}=\omega_{l}=\omega_{p} / 2\right)$ : the non-linear overlap of two counterpropagating modes yields a second harmonic field radiating from the upper surface of the waveguide. Because of its intrinsic higher produced signal, this interaction is well suited to provide a characterization of the effect of the added vertical microcavity and a quantitative estimation of the parametric gain.

The sample resulted by our numerical simulations was grown by metal organic chemical vapor deposition on (100) GaAs substrate. The planar structure was then chemically etched to create 2.5-3.5 $\mu \mathrm{m}$-thick ridges with 6-9 $\mu \mathrm{m}$ widths. The epitaxial structure consists in 4.5 period $\mathrm{Al}_{0.25} \mathrm{Ga}_{0.75} \mathrm{As} / \mathrm{Al}_{0.80} \mathrm{Ga}_{0.20} \mathrm{As} \mathrm{QPM}$ waveguide core, 41-period asymmetrical $\mathrm{Al}_{0.25} \mathrm{Ga}_{0.75} \mathrm{As} / \mathrm{Al}_{0.80} \mathrm{Ga}_{0.20} \mathrm{As} \mathrm{DBR}$ (serving as lower cladding + back mirror) and 18-period asymmetrical $\mathrm{Al}_{0.25} \mathrm{Ga}_{0.75} \mathrm{As} / \mathrm{Al}_{0.80} \mathrm{Ga}_{0.20} \mathrm{As} \mathrm{DBR}$ (serving as upper cladding + top mirror). Figure 5 reports the tuning curves calculated on the nominal structure.

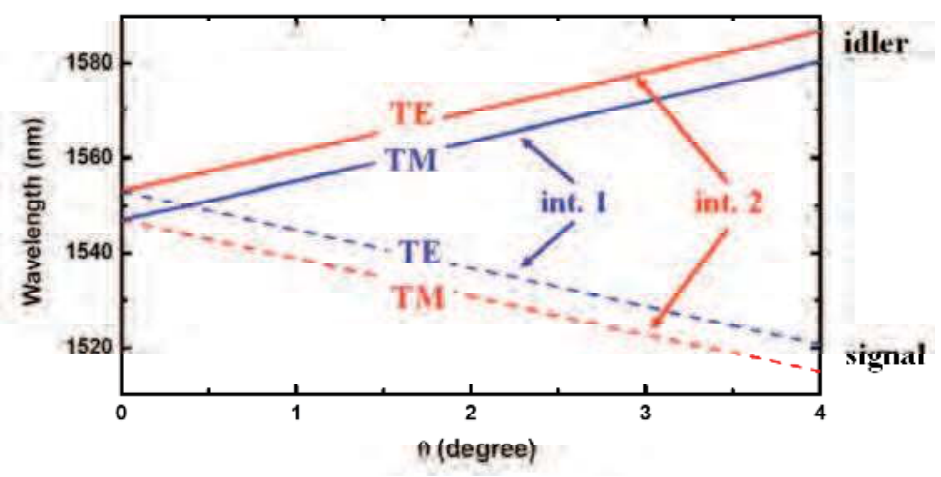

Fig. 5. Calculated tuning curves as a function of the pump incident angle for a pump wavelength of $775 \mathrm{~nm}$.

The SESHG measurements were performed employing one end-fire coupled fundamental frequency (FF) beam relying on Fresnel reflection at the opposite facet to obtain back propagating modes (see Fig. 6). 


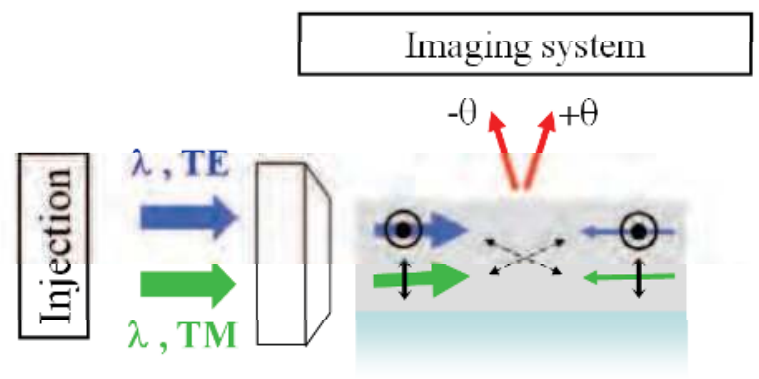

Fig. 6. Scheme of SESHG set up. TE and TM modes are end-fire excited through the same input facet, relying on their Fresnel reflection at the opposite facet. By doing so, two nonlinear processes occur: the interaction of the TE mode with the reflected part of the TM one, and the interaction of the TM mode with the reflected part of the TE one. This symmetric configuration results in the generation of two second harmonic waves of comparable amplitudes, which radiate with angles $\theta$ and $-\theta$, respectively: an interference pattern can thus be observed at the waveguide surface.

The fundamental frequency was linearly polarized at $45^{\circ}$ relative to the substrate so that the input power was equally divided between TE and TM eigenfields. The input beam was supplied by an external-cavity cw tunable laser beam, amplified with an $\mathrm{Er}^{3+}$-doped fiber. The SH field was acquired with an optical system mounted over the waveguide, perpendicularly to its plane. This consists of a CCD camera Bosch LTC 0335/50 1/3 inch format 512×582 pixels and an Edmund video lens trinocular VZM microscope for near field acquisition. In order to study the dependence of SESHG power vs the FF power, the generated signal was measured with a large area Si photodiode plus a lock-in synchronous detection.

The near-field image of the SH field obtained for a FF injected at $1.565 \mu \mathrm{m}$ is shown in Figure 7 ; we note that the difference between the resonance wavelength calculated for the nominal structure and the measured one is only $7,5 \mathrm{~nm}$ (which is totally compatible with the technological tolerances). The period $\Lambda$ of the pattern provides a straightforward measure of $\theta$ through the relation $|\sin \theta|=\lambda_{p} / 2 \Lambda$. In this case $\theta=0.4^{\circ}$ which is in excellent agreement with the value obtained by numerical predictions.

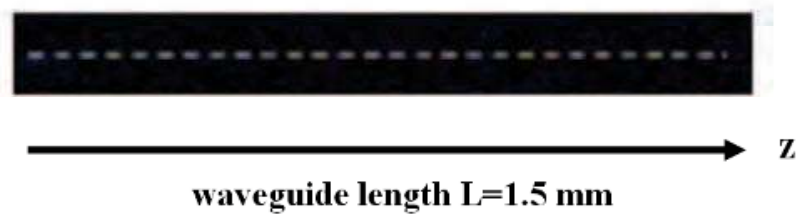

Fig. 7. Complete view of SESHG near field for a FF injected at $1.56 \mu \mathrm{m}$.

In Figure 8 the detected SESHG power is plotted versus the guided FF power: SESHG power data are in good agreement with the parabolic fit curve, as expected for a quadratic nonlinear process. 


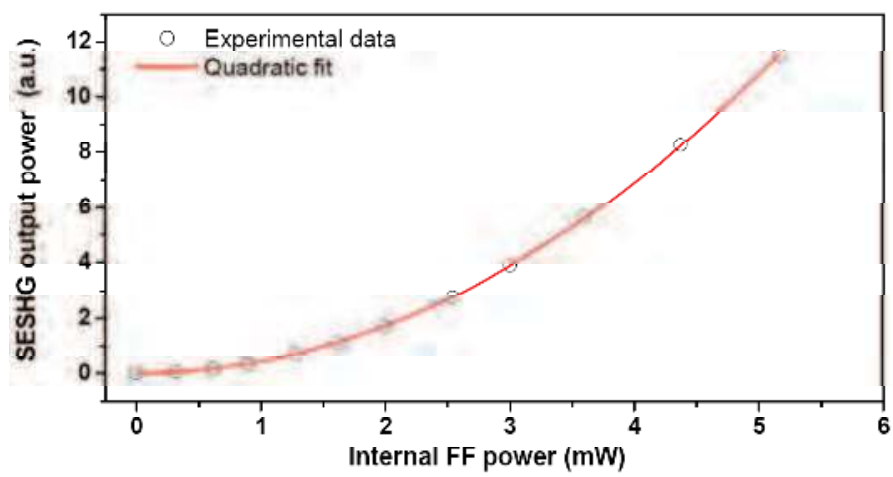

Fig. 8. SESHG power vs. FF power at $1.56 \mu \mathrm{m}$ (photodiode acquisition).

As we have pointed out, the net frequency dependence of the enhancement factor is dominated by the cavity; the spectrum of the second harmonic field allows thus the characterization of the effect of the integrated vertical cavity.

Figure 9 reports the experimental spectrum obtained with our sample; the experimental points are fitted with a Lorentzian, which is the expected theoretical shape close to the resonance wavelength. The calculated full width at half maximum of the Lorentzian, in the limit of perfect reflectivity of the bottom mirror, is:

$$
\gamma=\frac{T \lambda^{2}}{2 \pi n d}
$$

with $T$ the transmission coefficient of the upper mirror. The calculated value for our structure is $\gamma=0.55 \mathrm{~nm}$, which is in excellent agreement with the experimental data $(\gamma=0.54 \mathrm{~nm})$.

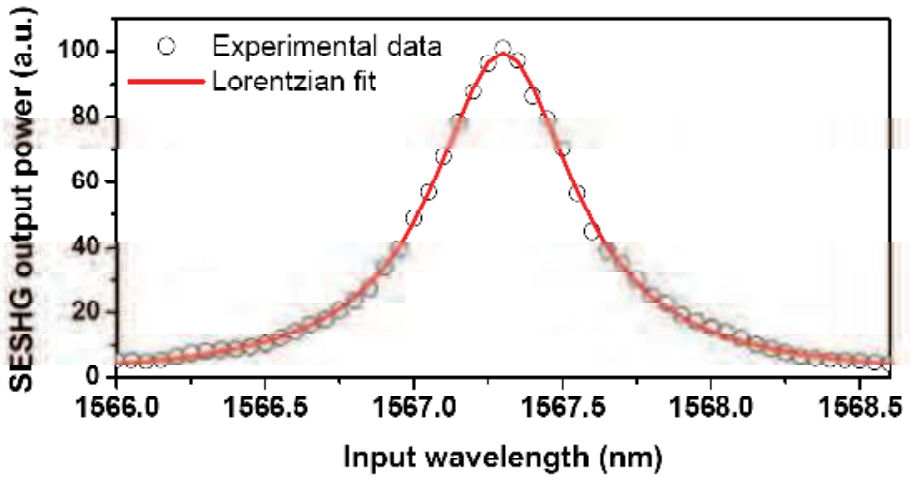

Fig. 9. Experimental spectrum of the SESHG signal (dots) and fit with a Lorentzian (solid line).

\subsection{Parametric fluorescence and coincidence histogram}

Since the sample described in the previous section presented elevated optical losses that have been imputed to the growth technique, a second sample was grown using the 
technique of molecular beam epitaxy. The epitaxial structure consists in 4.5 period $\mathrm{Al}_{0.25} \mathrm{Ga}_{0.75} \mathrm{As} / \mathrm{Al}_{0.80} \mathrm{Ga}_{0.20} \mathrm{As} \mathrm{QPM}$ waveguide core, 41-period asymmetrical $\mathrm{Al}_{0.25} \mathrm{Ga}_{0.75} \mathrm{As} /$ $\mathrm{Al}_{0.80} \mathrm{Ga}_{0.20} \mathrm{As}$ DBR (serving as lower cladding + back mirror) and 18-period asymmetrical $\mathrm{Al}_{0.25} \mathrm{Ga}_{0.75} \mathrm{As} / \mathrm{Al}_{0.80} \mathrm{Ga}_{0.20} \mathrm{As} \mathrm{DBR}$ (serving as upper cladding + top mirror).

Figure 10 shows our first parametric fluorescence spectrum; the pump beam is provided by a TE polarized, pulsed Ti: Sa laser with $\lambda_{p}=759,5 \mathrm{~nm}$ and a $3 \mathrm{kHz}$ repetition rate. The pulse peak power is $P_{p}=10 \mathrm{~W}$ and its duration is $150 \mathrm{~ns}$. The pump beam is focused on top of the waveguide ridge using a cylindrical lens with an angle $\theta$ in the $\mathrm{xz}$ plane. The generated photons are collected from one of the facets of the sample with a microscope objective, spectrally analyzed with a monochromator, and then coupled into a fibered InGaAs singlephoton avalanche photodiode (IdQuantique).

The spectra obtained for $\theta=3^{\circ}$, show the existence of the two expected processes (see Figure 5 ) and demonstrate the possibility of direct generation of polarization-entangled states. The amplitude difference in the observed signal is due to the fact that the long wavelength photons are collected after their reflection on the facet opposite to the detection. An antireflection coating to both facets of the sample would allow an automatic separation of the photons of each pair and their direct coupling into two optical fibers, through standard pigtailing process. The amount of detected photons allows deducing the brightness of our twin photon source, which we estimate around $10^{-11} \mathrm{~W}^{-1}$, which represents an improvement of at least two orders of magnitude with respect to (Lanco et al., 2006).

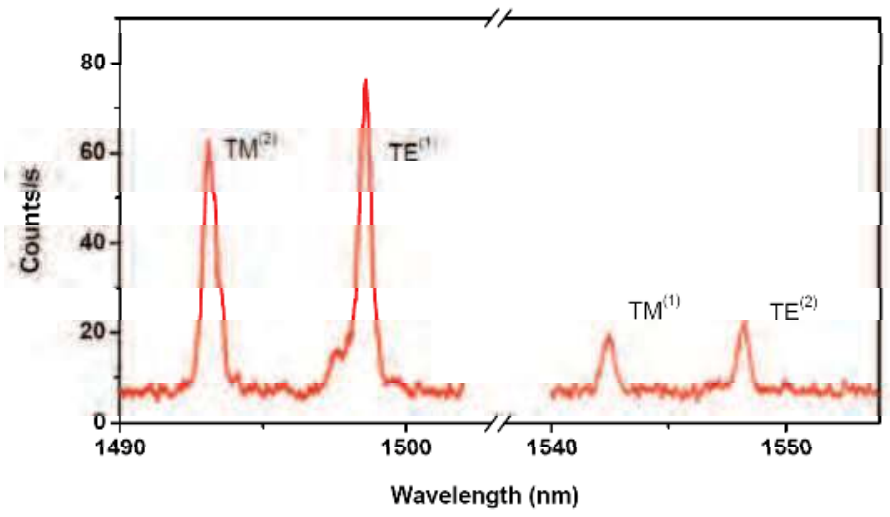

Fig. 10. Experimental spectrum of the parametric fluorescence for the two parametric interactions. The background noise here is due to the dark counts of the detectors.

To further assess the twin character of the emitted photons, the time correlations between the detected counts have been analyzed. The scheme of the setup is shown in Figure 11 and the histogram of the time delays is shown in Fig. 12, for the case of interaction 1 with $\theta=3^{\circ}$. With a sampling interval of 40 ps, the histogram results from an acquisition time of 25 minutes. The peak observed for $t_{s}=t_{i}$ demonstrates unambiguously the twin character of the generated photons; the 500 ps full width at half maximum of the histogram corresponds to the timing jitter of both detectors. The flat background is produced by the accidental coincidences essentially dues to dark counts: indeed, switching the pump polarization from TE to TM leads to the suppression of the $t_{s}=t_{i}$ peak, without modifying this background. Finally, no time-correlation is found between photons that are generated with different 
interactions: this agrees with the expectations, since these photons are not generated within the same nonlinear process.

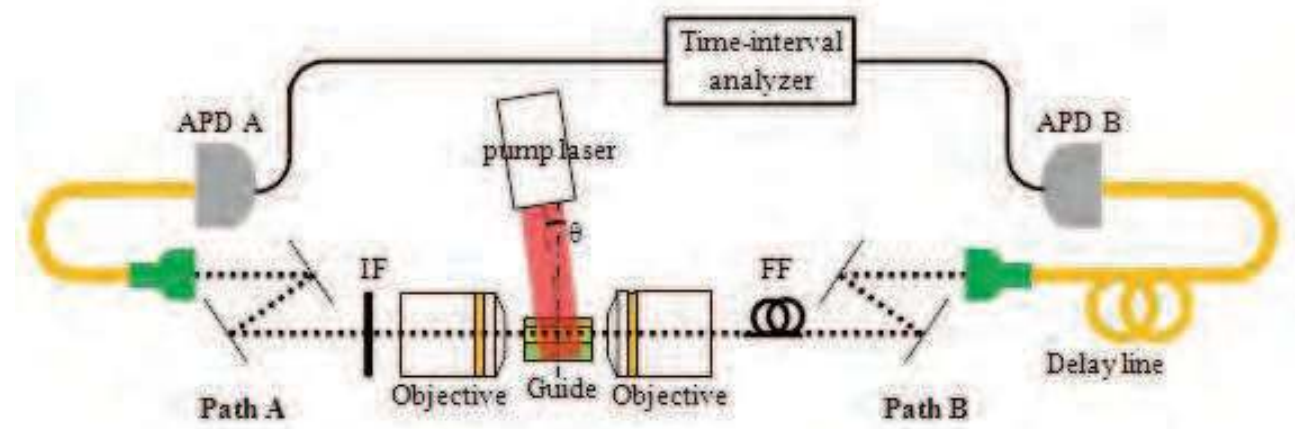

Fig. 11. Experimental set-up for the coincidence measurement. IF: interferential filter. FF: fibered filter. APD: single photon avalanche photodiode. The signal collected by the detectors is sent to a time-interval analyzer to built the time-correlation histogram.

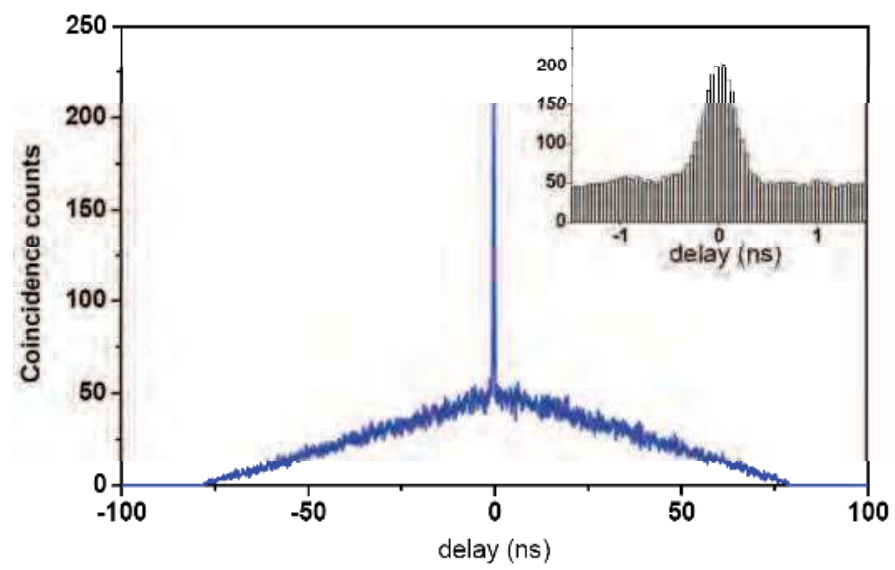

Fig. 12. Time-correlation histogram between counterpropagating photons.

\section{Conclusion and perspectives}

These results open the way to the demonstration of several interesting features associated to the counterpropagating geometry, as the direct generation of polarization entangled Bell state or the control of the generated two-photon state via an appropriate choice of the spatial and spectral pump beam profile. Indeed recent developments in quantum information theory have arisen a growing interest on 'generalized' states of frequency correlation (like frequency-correlated or frequency-uncorrelated photons). For example: i) quantum teleportation and entanglement swapping require the synchronized creation of multiple photon pairs, which is achieved by using a short pump pulse (thus relaxing the strict frequency anti-correlation of the generated photons mentioned above); ii) linear optical quantum computation requires uncorrelated photons in order to guarantee their 
indistinguishability; iii) improvements on clock synchronization need frequency correlation to overcome media dispersion.

The efficiency of this room temperature working device, along with the high-quality quantum properties of the generated photons and their telecom wavelength, makes this source a serious candidate for integrated quantum photonics.

\section{Acknowledgments}

The authors thank Isabelle Sagnes and Aristide Lemaître (Laboratoire de Photonique et Nanostructures CNRS UPR20, France) for sample growth and Pascal Filloux for ridge processing.

\section{References}

Andronico, A.; Caillet, X.; Favero, I.; Ducci, S. \& Berger, V. (2008). Semiconductor microcavities for enhanced nonlinear optics interactions. J. of European Optical Society - Rapid Publications, 3, (September 2008) (08030-1-08030-7), 1990-2573.

Aspect, A.; Grangier, P. \& Roger, G. (1982). Experimental Realization of Einstein-PodoskyRosen-Bohm Gedankenexperiment: A New Violation of Bell's Inequalities. Phys. Rev. Lett., 49, 2, (July 1982) (91-94), 0031-9007.

Banaszek, K.; U'Ren, A. B. \& Walmsley, I. A. (2001). Generation of correlated photons in controlled spatial modes by downconversion in nonlinear waveguides. Optics Letters, 26, 17, (September 2001) (1367-1369), 0146-9592.

Bouwmeester, D.; Pan, J.W.; Mattle, K.; Eibl, M.; Weinfurter, H. \& Zeilinger, A. (1997). Experimental quantum teleportation. Nature, 390, (December 1997) (575-579), 00280836.

Caillet, X.; Berger, V.; Leo, G.; Sagnes, I. \& Ducci, S. (2009). A semiconductor ridge micro cavity to generate counterpropagating twin photons, Proceedings of SPIE 7222 72221J, 0277-786X, pp. 72221J1-8, San Jose (CA, USA), January 2009, SPIE.

Deutsch, D. \& Ekert, A. (1998). Quantum Computation. Phys. World, 11, 3, (March 1998) (4752), 0953-8585.

Ducci, S.; Lanco, L.; Seurin, Y.; Leo, G.; Berger, V.; De Rossi, A. \& Marcadet, X. (2005). Semiconductor sources of twin photons for quantum information. J. of Optics B: Quantum and Semiclassical Optics, 7, (June 2005) (S158-S165), 1464-4266.

Fan, J. \& Migdall, A. (2007). A broadband high spectral brightness fiber-based two-photon source. Opt. Express, 15, 6, (March 2007) (2915-2920), 1094-4087.

Giovannetti, V.; Maccone, L.; Shapiro, J. H. \& Wong, F. N. C. (2002). Extended phasematching conditions for improved entanglement generation. Phys. Rev. A, 66, 4, (October 2002) (043813-1-043813-9), 1050-2947.

Gisin, N.; Ribordy, G.; Tittel, W. \& Zbinden, H. (2002). Rev. Mod. Phys. 74, 1, (March 2002) (145-195), 0034-6861.

Grice, W. P.; U'Ren, A. B. \& Walmsley, I. A. (2001). Eliminating frequency and space-time correlations in multiphoton states. Phys. Rev. A, 64, 6, (November 2001) (063815-1063815-7), 1050-2947. 
Kwiat, P. G.; Mattle, K.; Weinfurter, H.; Zeilinger, A.; Sergienko, A.V. \& Shih, Y. H. (1995). New High-Intensity Source of Polarization-Entangled Photon Pairs. Phys. Rev. Lett., 75, 24, (December 1995) (4337-4341), 0031-9007.

Lanco, L.; Ducci, S.; Likforman, J.-P.; Marcadet, X.; van Houwelingen, J. A. W.; Zbinden, H.; Leo, G. and Berger, V. (2006). Semiconductor Waveguide Source of Counterpropagating Twin Photons. Phys. Rev. Lett., 97, 17, (October 2006) (1739011-173901-4), 0031-9007.

Law, C. K.;Walmsley, I. A. \& Eberly, J. H. (2000) Continuous Frequency Entanglement: effective Finite Hilbert Space and Entropy Control. Phys. Rev. Lett. 84, 23, ( June 2000) (5304-5307), 0031-9007.

Migdall, A. (1999). Correlated-Photon Metrology Withiout Absolute Standards. Phys. Today, 52, 1 (January 1999) (41-46), 0031-9228.

Normandin, R. \& Stegeman, G. I. (1979). Nondegenerate four-wave mixing in integrated optics. Opt. Lett., 4, 58 (February 1979) (58-59), 0146-9592.

Perina, J. (2008). Quantum properties of counterpropagating two-photon states generated in a planar waveguide. Phys. Rev. A, 77, 1, (January 2008) (013803-1-013803-14), 10502947.

Rarity, J. G.; Fulconis, J.; Duligall, J.; Wadsworth, W. J. \& Russel, P. S. J. (2005). Photonic crystal fiber source of correlated photon pairs. Opt. Express, 13, 2, (January 2005) (534-544), 1094-4087.

Sergienko, A.V. \& Jaeger, G.S. (2003). Quantum information processing and precise optical measurement with entangled-photon pairs. Contemporary Phys., 44, 4, (July 2003) (341-356), 0010-7514.

Stevenson, R. M.; Young, R. J.; Atkinson, P.; Cooper, K.; Ritchie, D. A. \& Shields, A. J. (2006). A semiconductor source of triggered entangled photon pairs. Nature, 439, (January 2006) (179-182), 0028-0836.

Tanzilli, S.; De Riedmatten, H.; Tittel, W.; Zbinden, H.; Baldi, P.; De Micheli, M.; Ostrowsky, D. B. \& Gisin, N. (2001). Highly efficient photon-pair source using a Periodically Poled Lithium Niobate waveguide. Electronics Letters, 37, 1, (January 2001) (26-28), 0013-5194.

Tittel, W.; Brendel, J.; Zbinden, H. \& Gisin, N. (1998). Violation of Bell Inequalities by Photons More Than 10 km Apart. Phys. Rev. Lett., 81, 17, (October 1998) (3563-3566), 0031-9007.

Torres, J. P.; Macia, F.; Carrasco, S. \& Torner, L. (2005). Engineering the frequency correlations of entangled two-photon states by achromatic phase matching. Optics Letters, 30, 3, (February 2005) (314-316), 0146-9592.

Walther, P.; Resch, K. J.; Rudolf, T.; Schenck, E.; Weinfurter, H.; Vedral, V.; Aspelmeyer, M. \& Zeilinger, A. (2005). Experimental one-way quantum computing. Nature, 434, (March 2005) (169-176), 0028-0836.

Walton, Z. D.; Booth, M. C.; Sergienko, A. V.; Saleh B. E. A. \& Teich, M. C. (2003). Controllable frequency entanglement via auto-phase-matched spontaneous parametric down-conversion. Phys. Rev. A, 67, 5 (May 2003) (053810-1-053810-4), 1050-2947. 
Walton, Z. D.; Sergienko, A. V.; Saleh, B. E. A. \& Teich, M. C. (2004). Generation of polarization-entangled photon pairs with arbitrary joint spectrum. Phys. Rev. A, 70, 5, (November 2004) (052317-1-052317-5), 1050-2947.

Wang, L. J.; Hong, C. K. \& Friberg, S. R. (2001). Generation of correlated photons via fourwave mixing in optical fibres. J. Opt. B: Quantum and Semiclass. Opt., 3, (October 2001) (346-352), 1464-4266.

Weihs, G.; Jennewein, T.; Simon, C.; Weinfurter, H. \& Zeilinger, A. (1998). Violation of Bell's Inequality under Strict Einstein Locality Conditions. Phys. Rev. Lett., 81, 23, (December 1998) (5039-5043), 0031-9007. 


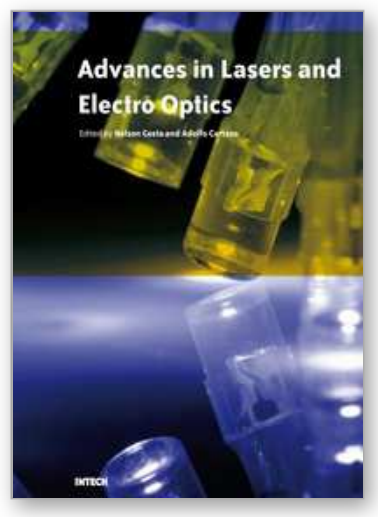

\author{
Advances in Lasers and Electro Optics \\ Edited by Nelson Costa and Adolfo Cartaxo
}

ISBN 978-953-307-088-9

Hard cover, 838 pages

Publisher InTech

Published online 01, April, 2010

Published in print edition April, 2010

Lasers and electro-optics is a field of research leading to constant breakthroughs. Indeed, tremendous advances have occurred in optical components and systems since the invention of laser in the late $50 \mathrm{~s}$, with applications in almost every imaginable field of science including control, astronomy, medicine, communications, measurements, etc. If we focus on lasers, for example, we find applications in quite different areas. We find lasers, for instance, in industry, emitting power level of several tens of kilowatts for welding and cutting; in medical applications, emitting power levels from few milliwatt to tens of Watt for various types of surgeries; and in optical fibre telecommunication systems, emitting power levels of the order of one milliwatt. This book is divided in four sections. The book presents several physical effects and properties of materials used in lasers and electro-optics in the first chapter and, in the three remaining chapters, applications of lasers and electro-optics in three different areas are presented.

\title{
How to reference
}

In order to correctly reference this scholarly work, feel free to copy and paste the following:

Xavier Caillet, Adeline Orieux, Ivan Favero, Giuseppe Leo and Sara Ducci (2010). Semiconductor Ridge Microcavities Generating Counterpropagating Entangled Photons, Advances in Lasers and Electro Optics, Nelson Costa and Adolfo Cartaxo (Ed.), ISBN: 978-953-307-088-9, InTech, Available from: http://www.intechopen.com/books/advances-in-lasers-and-electro-optics/semiconductor-ridge-microcavitiesgenerating-counterpropagating-entangled-photons

\section{INTECH}

open science | open minds

\section{InTech Europe}

University Campus STeP Ri

Slavka Krautzeka 83/A

51000 Rijeka, Croatia

Phone: +385 (51) 770447

Fax: +385 (51) 686166

www.intechopen.com

\section{InTech China}

Unit 405, Office Block, Hotel Equatorial Shanghai

No.65, Yan An Road (West), Shanghai, 200040, China

中国上海市延安西路 65 号上海国际贵都大饭店办公楼 405 单元

Phone: +86-21-62489820

Fax: $+86-21-62489821$ 
(C) 2010 The Author(s). Licensee IntechOpen. This chapter is distributed under the terms of the Creative Commons Attribution-NonCommercialShareAlike-3.0 License, which permits use, distribution and reproduction for non-commercial purposes, provided the original is properly cited and derivative works building on this content are distributed under the same license. 